\title{
High-temperature poling of ferroelectrics
}

\author{
Alain B. Kounga, ${ }^{\text {a) }}$ Torsten Granzow, ${ }^{\text {b) }}$ Emil Aulbach, Manuel Hinterstein, and \\ Jürgen Rödel \\ Institute of Materials Science, Technische Universität Darmstadt, Petersenstrasse 23, 64287 Darmstadt, \\ Germany
}

(Received 9 January 2008; accepted 27 May 2008; published online 30 July 2008)

\begin{abstract}
The poling behavior of a lead-zirconate-titanate piezoelectric ceramic is investigated by measurements of the ferroelectric hysteresis, the longitudinal piezoelectric coefficient, and field-cooling poling experiments. At high temperatures, the decrease in the coercive field facilitates poling at lower electric fields, resulting in higher values of the longitudinal piezoelectric coefficient. However, there exists a threshold field of about $150 \mathrm{~V} / \mathrm{mm}$, below which fully poled samples cannot be obtained even when field cooling from temperatures above the transition. Further, a temperature regime below the Curie temperature is observed, where a polarization under field can be measured, but a remanent polarization is not stable. The results are discussed with respect to the phase transition behavior. (C) 2008 American Institute of Physics. [DOI: 10.1063/1.2959830]
\end{abstract}

\section{INTRODUCTION}

Piezoelectric ceramics such as lead zirconate titanate (PZT) or comparable materials with perovskite structure can be found in a multitude of applications, ranging from stress sensors and high-frequency microphones to ultrasound emitters, medical injectors, and large-strain actuators. ${ }^{1-3}$ Most of these applications require electrical poling, i.e., there must be a preferred orientation of the ferroelectric domains that goes along with a macroscopic polarization. Traditionally, the major objective in all poling stages is to use the lowest possible electric field for the shortest possible time to induce the maximum degree of domain alignment at a temperature at which the achieved polarization is stable. ${ }^{4-11}$ Application of high fields for an extended time can introduce an electrical bias field and an offset polarization in the sample that is caused not by the reorientation of domains but by the redistribution of mobile charge carriers. ${ }^{12}$ This contribution to the total polarization is hard to control and thus to be avoided. The exact nature of the optimum ferroelectric domain structure, however, depends on the particular application for which the material is intended. As an example, a piezoelectric sensor needs a high degree of domain orientation and a stable domain structure to guarantee a linear response in a wide stress range, while an actuator can benefit from a less stable domain structure that allows large-scale non- $180^{\circ}$ domain switching associated with high strain. Obviously, a controlled poling procedure is of paramount importance to obtain optimum material performance. For some materials, however, poling is quite difficult. This is especially true for most newly developed piezoelectrics such as hightemperature piezoceramics or lead-free materials. These materials often suffer from high electrical conductivity, high coercive field, or low breakdown strength; thus, it is sometimes difficult to apply a field high enough to obtain any

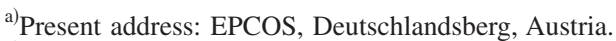

b) Author to whom correspondence should be addressed. Tel.:+49 6151 166317. FAX: +49 6151 166314. Electronic mail: granzow@ceramics.tudarmstadt.de.
}

preferred domain orientation at all. To remedy some of these problems, it has been demonstrated in previous communications that the minimum electric field necessary for poling can be reduced by applying a transverse mechanical load during poling. ${ }^{13,14}$ This may represent an advantage when it comes to poling ceramic compositions with very high coercive fields.

Similarly, piezoelectric materials such as acceptor doped PZT are very hard to pole at room temperature (RT) due to their intrinsic aging behavior. Many manufacturers utilize a poling procedure optimized for their specific material and application, and many of these procedures include heating of the sample since domain mobility, which is known to play an important role in the poling process, generally increases with increasing temperature. ${ }^{15-18}$ However, a systematic investigation on the relation between poling temperature (including the temperature regime around the Curie temperature), poling field, polarization, and the component $d_{33}$ of the piezoelectric tensor is missing so far. We remedy this deficiency by examining the development of the piezoelectric properties and ferroelectric polarization using different poling procedures. The results are correlated with structural data obtained from x-ray diffraction (XRD) measurements to investigate the influence of the paraelectric-ferroelectric phase transition. The results afford a better understanding of the poling process at temperatures both above and below the Curie point of ferroelectric ceramics.

\section{EXPERIMENTAL METHODS}

The present investigation was carried out using a commercially available PIC 151 (PI Ceramics, Lederhose, Germany), a soft PZT with the composition near the morphotropic phase boundary, $\mathrm{Pb}_{0.99}\left[\mathrm{Zr}_{0.45} \mathrm{Ti}_{0.47}\left(\mathrm{Ni}_{0.33} \mathrm{Sb}_{0.67}\right)_{0.08}\right] \mathrm{O}_{3}$. According to the manufacturer, the material undergoes a paraelectric (cubic) $\rightarrow$ ferroelectric (tetragonal or rhombohedral) phase transition at the Curie temperature $T_{C}=250{ }^{\circ} \mathrm{C}$. At RT, the spontaneous polarization is $P_{s} \approx 40 \mu \mathrm{C} / \mathrm{cm}^{2}$ and the coercive field is $E_{c} \approx 1 \mathrm{kV} / \mathrm{mm}$. The lattice parameters 
and phase compositions were analyzed by powder XRD measurements at different temperatures between RT and $500{ }^{\circ} \mathrm{C}$ at the experimental station B2 (Ref. 19) in the synchrotron laboratory HASYLAB at DESY (Hamburg, Germany). All measurements were performed in the DebyeScherrer geometry in a capillary using an on-site readable image plate detector OBI (Ref. 20) and a STOE furnace. For the high-temperature measurements, $\lambda=0.48918 \AA$ was selected. A full profile Rietveld structure refinement was performed within the GSAS program package. ${ }^{21}$

Between RT and $300{ }^{\circ} \mathrm{C}$, the two-phase model of a tetragonal $P 4 \mathrm{~mm}$ and a monoclinic $\mathrm{Cm}$ phase was used. In PZT samples of morphotropic composition, nanodomain structures can directly be linked to the detection of intensity previously interpreted as a monoclinic phase but are not necessarily of monoclinic internal symmetry. ${ }^{22}$ Between 320 and $500{ }^{\circ} \mathrm{C}$, a single phase model with cubic $\operatorname{Pm} 3 \mathrm{~m}$ structure was used. The phase transition point was determined by analyzing the pseudocubic $\{200\}_{c}$ reflections and the overall $\chi^{2}$ of the refinement.

For the electrical measurements, cylindrical samples with a diameter of $10 \mathrm{~mm}$ and a thickness of $1 \mathrm{~mm}$ were used. Both circular faces were electroded using silver paste. The experimental setup for high-temperature electrical measurement consisted of a split furnace with Kanthal heating wires and a type $K$ thermocouple (Thermal Technologies). The sample was placed in the immediate vicinity of the temperature sensor between two thin $\mathrm{Al}_{2} \mathrm{O}_{3}$ ceramic tubes and contacted with Pt wire. After heating the sample to a target temperature, a dwell time of 10 min was allowed for thermal equilibration. Different types of measurements were performed. One was the measurement of the polarization hysteresis at different temperatures using a modified SawyerTower bridge. In the second set of measurements, the samples were field cooled, i.e., the sample was heated to the starting temperature $T_{\text {start }}$ and an electric field $E_{\mathrm{fc}}$ was applied and then kept constant while the sample was cooled to RT with a cooling rate of $6{ }^{\circ} \mathrm{C} / \mathrm{min}$. Once at RT, the electric field was removed and the piezoelectric coefficient $d_{33}$ was measured using a Berlincourt meter (YE 2730 Sinocera, Shanghai, China) according to IEEE standards. ${ }^{23}$

As the remnant polarization is one of the decisive factors that determine $d_{33}$, the development of the polarization when poling at high temperatures was investigated in the third set of measurements. The aim was to find an optimum poling temperature for a given electric field, defined by maximum values of the polarization. As the electrical conductivity of the samples at $T>200{ }^{\circ} \mathrm{C}$ was too high to determine $P_{\text {rem }}$ or $P_{\max }$ from hysteresis measurements, a different measurement method that allowed for compensation of the electrical current through the sample had to be employed. The sample was heated to $370{ }^{\circ} \mathrm{C}$, well above the Curie temperature, to guarantee complete depoling. It was then cooled to a specific temperature $300{ }^{\circ} \mathrm{C}>T>200{ }^{\circ} \mathrm{C}$, and an electric field of $400 \mathrm{~V} / \mathrm{mm}$ was applied for $5 \mathrm{~min}$. The charge $Q$ accumulating on the measuring capacitance resulted both from the polarization change in the sample and the electrical current through the sample. Polarization dynamics ceased within several tens of seconds, and after $5 \mathrm{~min}$, the $Q(t)$ curve was
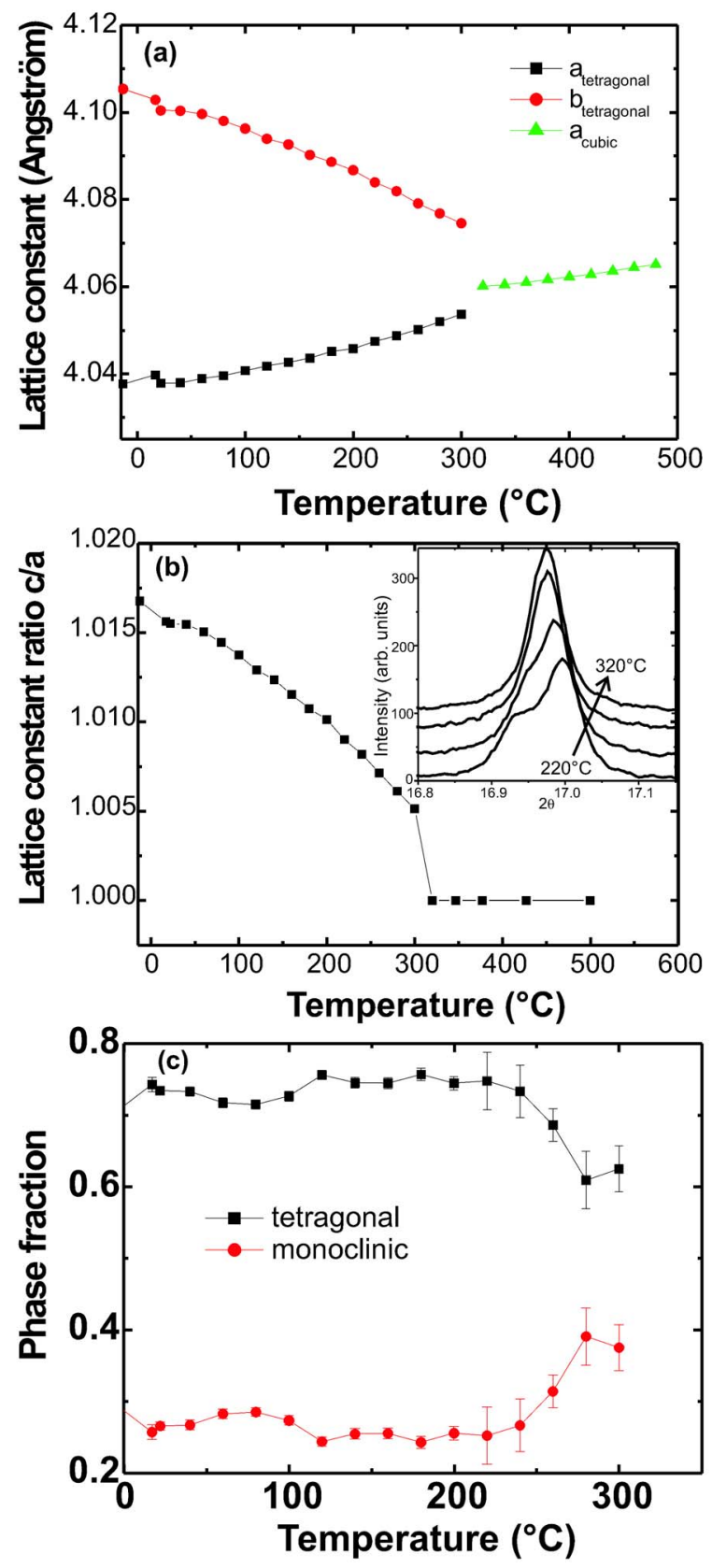

FIG. 1. (Color online) Structure analysis of the material studied. (a) Temperature dependence of lattice parameters, (b) $c / a$ ratio, and (c) phase fraction of tetragonal and monoclinic structures. The inset of (b) shows the pseudocubic $\{200\}_{c}$ reflection at $220,260,300$, and $320^{\circ} \mathrm{C}$.

linear, indicating a constant Ohmic current through the sample. Linear extrapolation of $Q(t)$ to $t=0$ gave the value of the maximum polarization $P_{\max }$. The field was then turned off, and the polarization dropped back to the value of $P_{\text {rem }}$. A similar procedure is described, e.g., in Ref. 24. The sample was then heated to $370{ }^{\circ} \mathrm{C}$ again for complete depoling, and the procedure was repeated at a lower temperature.

\section{RESULTS}

The lattice constant and the phase fraction resulting from the XRD measurements are shown in Fig. 1. The phase transition from the paraelectric cubic high-temperature to the ferroelectric low-temperature phase is very clearly observed 

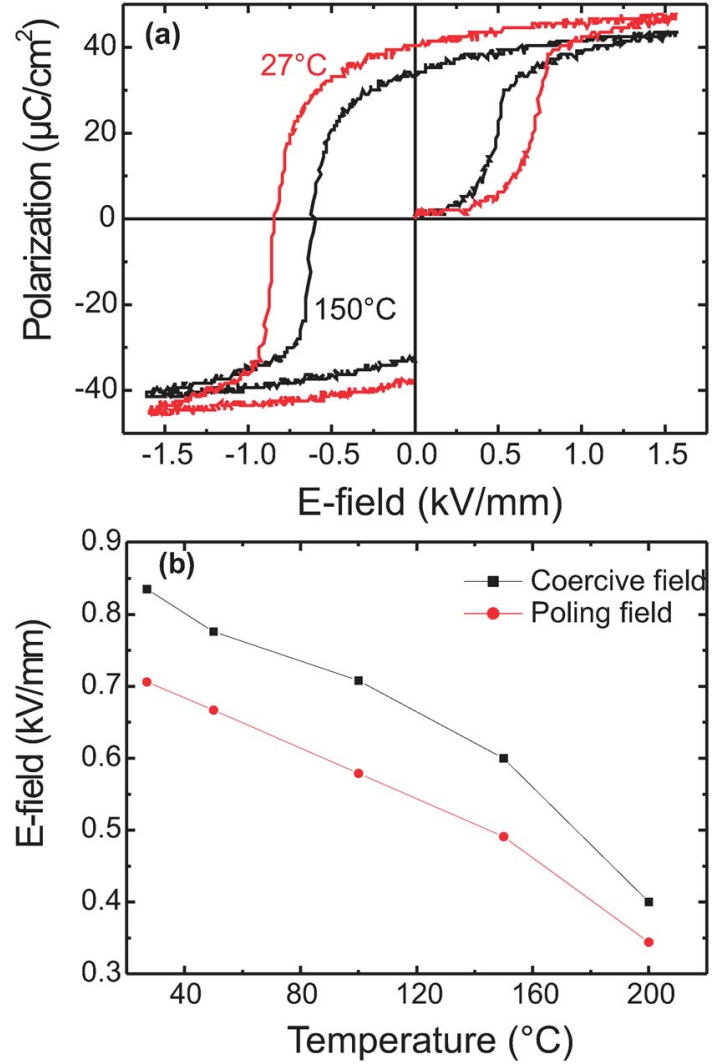

FIG. 2. (Color online) (a) Polarization hysteresis at RT and $150^{\circ}$ Temperature-dependent coercive and poling fields.

in the splitting of the lattice constants, as provided in Fig. $1(\mathrm{a})$, and likewise in the splitting of the pseudocubic $\{200\}_{c}$ reflections. An asymmetry can be observed up to $300{ }^{\circ} \mathrm{C}$, implying a tetragonal distortion of the unit cell. Thus the Curie temperature $T_{C}$ lies between 320 and $300{ }^{\circ} \mathrm{C}$. When cooling from 320 to $300{ }^{\circ} \mathrm{C}$, the $\mathrm{c} / \mathrm{a}$ ratio jumps from unity for the cubic phase to values around 1.005 then continues to increase steadily upon further cooling to RT, as shown in Fig. 1(b). The inset of Fig. 1(b) exemplarily shows the pseudocubic $\{200\}_{c}$ reflections at $220,260,300$, and $320{ }^{\circ} \mathrm{C}$. Lattice constants were obtained by assuming a transition from a purely cubic to a mixed tetragonal and monoclinic structure. This is not entirely correct, as the material shows a first order phase transition, and therefore a coexistence region where all three phases are present. Full pattern Rietveld analysis of the diffraction patterns yielded the phase fractions, as displayed in Fig. 1(c). Between 300 and $250{ }^{\circ} \mathrm{C}$, the phase fraction of the tetragonal to monoclinic ratio increases continuously to about 3:1 and maintains this value down to lower temperatures.

Figure 2(a) exemplarily depicts the $P(E)$-hysteresis loops at RT and at $150{ }^{\circ} \mathrm{C}$. Since a fresh, unpoled sample was used for each measurement, all $P(E)$ curves start from zero. Thermodynamic theory predicts that the spontaneous polarization and coercive field decrease with increasing temperature. ${ }^{25}$ Correspondingly, the curves get slimmer and smaller at higher temperatures. This can be quantified by taking the values of the poling field $E_{p}$, defined as the inflection point of the $P(E)$ curve during the first poling, ${ }^{13}$ the
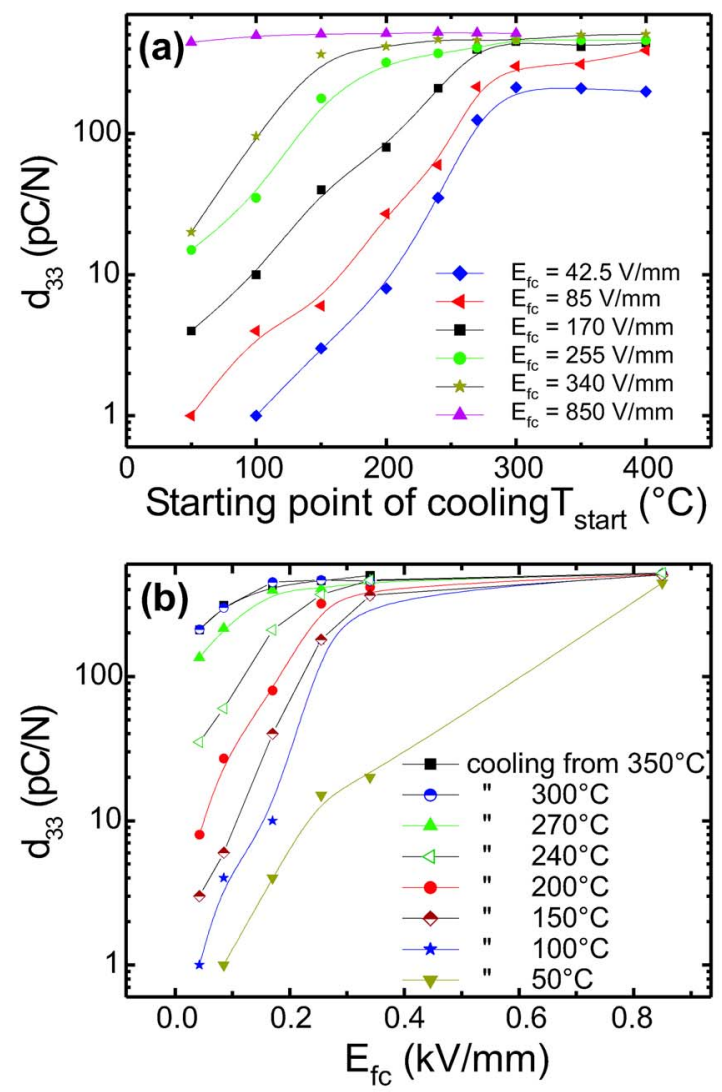

FIG. 3. (Color online) (a) Piezoelectric coefficient $d_{33}$ for different poling fields after field cooling as a function of starting temperature. (b) $d_{33}$ for different starting temperatures as a function of the poling field.

maximum polarization $P_{\max }$ at the highest field values, the remnant polarization $P_{\text {rem }}$ at zero field, and the coercive field $E_{c}$ where $P(E)$ crosses the $x$ axis. Figure 2(b) indicates that $E_{p}$ decreases from 0.7 to about $0.35 \mathrm{kV} / \mathrm{mm}$, whereas $E_{c}$ decreases from 0.85 to approximately $0.4 \mathrm{kV} / \mathrm{mm}$ in a temperature interval ranging from RT to $200{ }^{\circ} \mathrm{C}$. The hysteresis curve could not be measured reliably at higher temperatures due to strongly increasing leakage currents.

A field-cooling experiment was performed to investigate the influence of the poling temperature on the piezoelectric performance. A sample was heated to a starting temperature $T_{\text {start }}$ between 400 and $50{ }^{\circ} \mathrm{C}$. At $T_{\text {start }}$, an electric field $E_{\mathrm{fc}}$ was applied to the sample, and the sample was cooled to RT before the field was turned off. For this set of measurements, electric fields of $42.5,85,170,255,340$, and $850 \mathrm{~V} / \mathrm{mm}$ were chosen, corresponding to $5 \%, 10 \%, 20 \%, 30 \%, 40 \%$, and $100 \%$ of $E_{c}$ at RT. After field cooling, the piezoelectric coefficient $d_{33}$ of each sample was measured at RT. Results are presented in Fig. 3 in two different ways. Figure 3(a) depicts $d_{33}$ as a function of $T_{\text {start }}$ for the six different poling fields $E_{\mathrm{fc}}$. Figure $3(\mathrm{~b})$ provides the same data, but here $d_{33}$ is plotted as a function of $E_{\mathrm{fc}}$ for eight different starting temperatures $T_{\text {start }}$. Note that in both cases, the $d_{33}$ axis is presented in a logarithmic scale.

All curves in both figures clearly display two different regions. For low $T_{\text {start }}$ [Fig. 3(a)] or low $E_{\text {fc }}$ [Fig. 3(b)], an increase in $d_{33}$ is observed before the curves reach saturation at higher $T_{\text {start }}$ and $E_{\mathrm{fc}}$, respectively. Figure 3(a) shows the 


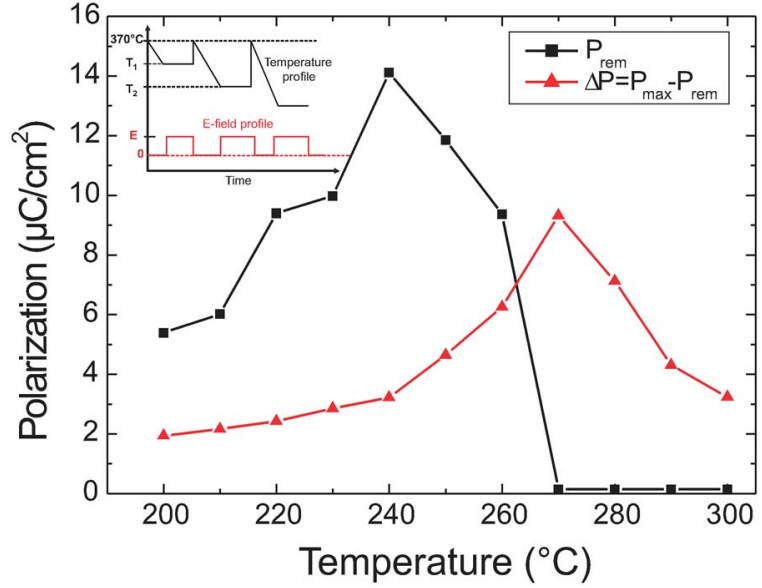

FIG. 4. (Color online) Temperature-dependent remnant polarization $P_{\text {rem }}$ and $\Delta P=P_{\max }-P_{\text {rem }}$ obtained by the intermittent poling procedure under an applied field of $400 \mathrm{~V} / \mathrm{mm}$. The inset shows the temporal changes in temperature and electric field.

$T_{\text {start }}$ where saturation is reached depending on $E_{\mathrm{fc}}$ : at the highest poling field, $d_{33}$ is nearly independent of $T_{\text {start }}$, while for the lowest poling field, $d_{33}$ reaches saturation only for $T_{\text {start }}>300{ }^{\circ} \mathrm{C}$. Note that for $E_{\mathrm{fc}}<170 \mathrm{~V} / \mathrm{mm}$, the saturation value of $d_{33}\left(T_{\text {start }}\right)$ is considerably lower than that for higher fields. This is also clearly visible in Fig. 3(b). Apparently, the ceramics cannot be fully poled with arbitrarily small fields even when field cooling starts from the paraelectric phase.

To investigate the relation between the inability to reach maximum $d_{33}$ values with low fields and the domain stability at high temperatures, we measured the development of polarization under field at high temperatures directly. The sample was heated to $370{ }^{\circ} \mathrm{C}$ to remove all traces of remnant polarization, then cooled to a certain poling temperature $T_{\text {pol }}$. Subsequently a field of $400 \mathrm{~V} / \mathrm{mm}$ was applied to the sample, and the resulting change in surface charge $Q$ was recorded. The total surface charge is a result of displacement current due to changes in the polarization and leakage current due to the drift of charge carriers through the sample. After the displacement current has subsided, the constant leakage current leads to a linear increase in the surface charge with time. Extrapolating the linear part of the $Q(t)$ curve to $t=0$ gives the fraction of the charge induced by the displacement current, which is proportional to the maximum polarization under field, $P_{\max }\left(T_{\text {pol }}\right)$. The field was then switched off, and the polarization quickly dropped back by the value $\Delta P$ to the remnant polarization $P_{\text {rem }}$. The sample was then depolarized by heating to $370{ }^{\circ} \mathrm{C}$ again, and the measurement was repeated at a lower poling temperature $T_{\text {pol }}$. Figure 4 shows $P_{\text {rem }}$ and the difference $\Delta P=P_{\max }-P_{\text {rem }}$ as functions of $T_{\text {pol }}$. The inset schematically displays the change in temperature and external electric field with time. Apparently, a stable polarization cannot be induced at high temperatures, as the values of $P_{\text {rem }}$ are nearly negligible for $T_{\text {pol }}>260{ }^{\circ} \mathrm{C}$. The high values of $\Delta P$ indicate that a noticeable polarization can be reached with an applied field even at $T_{\mathrm{pol}}=300{ }^{\circ} \mathrm{C}$; however, this polarization is not stable and vanishes when the field is removed. At $T_{\text {pol }} \leq 260{ }^{\circ} \mathrm{C}, P_{\text {rem }}$ increases quickly and reaches a maximum at $240{ }^{\circ} \mathrm{C}$ before it decreases again at even lower temperatures. Simultaneously, $\Delta P$ shows a steady decrease.

\section{DISCUSSION}

Both the XRD measurements and the hysteresis measurements are in agreement with known facts from the phase diagram of PZT and expectations of the typical behavior of ferroelectrics at increasing temperature. The phase transition from the paraelectric cubic to the ferroelectric phase is clearly demonstrated by the diffraction data. $T_{C}$ is determined to lie between 300 and $320{ }^{\circ} \mathrm{C}$, higher than the value of $250{ }^{\circ} \mathrm{C}$ given by the manufacturers. The change in phase fraction between 250 and $300{ }^{\circ} \mathrm{C}$ in favor of the monoclinic phase marks the point where the pseudocubic $\{200\}_{c}$ reflections remain the only evidence for tetragonal splitting. Considering the fact that remnant poling is not possible for temperatures higher than $260{ }^{\circ} \mathrm{C}$, there may be a coexistence of cubic and tetragonal phases in this temperature region. Some coherent scattering noncubic fractions remain, and the cubic and tetragonal mixture can be described best with a monoclinic model. The $c / a$ ratio of the tetragonal phase shows a $\sqrt{2}$ dependence in temperature evolution, equivalent to the behavior of an order parameter characteristic of the phase transition with $T_{C}$ between 300 and $320{ }^{\circ} \mathrm{C}$. The increase in $c / a$ at lower temperatures is one point that has to be taken into account when discussing the high-temperature poling behavior as well as the notable shift in the phase composition toward the tetragonal phase for $T<260{ }^{\circ} \mathrm{C}$. The decrease in $P_{\text {rem }}$ and $P_{\max }$ as well as poling and coercive field with increasing temperature is in agreement with simple thermodynamic considerations.

Figure 3 demonstrates that field-cooling ferroelectrics from temperatures above $T_{C}$ yields high $d_{33}$ values even under relatively low field exposure. Depending on the applied field, the results indicate two distinct regions: a region in which $d_{33}$ is sensitive to the starting cooling temperature and a plateau region in which the obtained piezoelectric coefficient does not depend on the starting temperature. Obviously, even a rather low symmetry-breaking external electric field leads to the formation of a stable preferred domain orientation when field cooling through the phase transition. Nearsaturation values are achieved when field cooling starts from a temperature where the coercive field, as determined by hysteresis measurements, is lower than the applied field. However, there seems to be a mechanism that prevents poling with very low fields; apparently, there is a threshold field of about $150 \mathrm{~V} / \mathrm{mm}$ below which only partial poling is achieved. In other words, the coercive field of the system never drops below $150 \mathrm{~V} / \mathrm{mm}$ independent of temperature but rather levels off at this value as the transition temperature is reached and then vanishes completely in the paraelectric phase. This is taken as an indication that the transition is of first order: the spontaneous polarization and, consequently, the spontaneous strain do not increase continuously from zero during poling but jump to a certain finite value. At the boundaries of areas with homogeneous spontaneous polarization and strain, depolarizing fields and stresses induced by 
polarization and strain mismatch can occur. Within one grain of the ceramic, the polarization and strain mismatch between domains can be compensated for by the formation of a local domain structure, but between different grains with different crystallographic orientations, the mismatch cannot be compensated for. If the applied field is too low, it is not sufficient to overcome the depolarizing fields and stresses in some regions, and a preferred polarization orientation cannot appear in these regions. The total polarization and thus the piezoelectric coefficient therefore do not reach the values of the fully poled sample. The effect is more pronounced in ceramics than in single crystals where no mismatch between grains can occur and all fields and stresses can be compensated for by the domain structure. If the transition is of second order, the interfacial depolarizing effects would be arbitrarily small at the transition point, and thus arbitrarily low fields would be sufficient for full poling.

This energy threshold for the appearance of a preferred domain orientation also gives an explanation for some of the peculiar features of $P_{\text {rem }}$ and the domain stability seen in Fig. 4. It is remarkable that $P_{\text {rem }}$ remains negligible upon cooling until $T=260{ }^{\circ} \mathrm{C}$ is reached, although the XRD data clearly identify the material as ferroelectric. $P_{\max }$ and $\Delta P$ are quite large, indicating the presence of noncentrosymmetric areas in the sample, which cause a large polarization under field and thus a high electrical susceptibility of about $\chi=\Delta P / E_{\mathrm{pol}}$ $=25000$. However, this polarization is not stable and decays as soon as the field is removed. The mismatch-induced depolarizing fields and stresses between grains are a possible driving force for this polarization decay. ${ }^{26}$ It is noteworthy that the final appearance of a stable remnant polarization coincides with the increase in the tetragonal phase fraction as seen in the XRD data. Apparently, tetragonal domains are more easily oriented and result in less mismatch of polarization and strain than a mixture of different structures. This would be in agreement with the observation that the application of an electric field at high temperatures leads to an increase in the tetragonal phase fraction. ${ }^{27}$ If the domain structure is not predominantly tetragonal, application of an external field will increase the fraction of tetragonal domains, but this change is not stable. When the field is removed, the structure reverts to its original distribution, and the polarization is destroyed. Only when the phase content is already largely tetragonal can the applied field result in a stable domain structure and a notable remnant polarization.

The inability of the material to keep a permanent polarization at temperatures above $260{ }^{\circ} \mathrm{C}$ is also an explanation for the different $T_{\mathrm{C}}$ values reported by the manufacturer and observed in our XRD data. The manufacturer determined $T_{\mathrm{C}}=250{ }^{\circ} \mathrm{C}$ by measuring the dielectric susceptibility, a method that integrates over the entire sample and is thus susceptible only to the long-range order of ferroelectric domains. Due to the depolarizing effects at grain boundaries, the long-range order breaks down before the local distortions of the unit cells disappear; thus, the material appears paraelectric. In the temperature range between 260 and $300{ }^{\circ} \mathrm{C}$, XRD still evidences the local distortion, which can result in ordered structures on the scale of several tens of nanometers, thus correctly identifying the phase as ferroelec- tric, while the macroscopic measurements are sensitive to the vanishing remnant polarization only, thus making the material appear paraelectric.

It should be noted that parameters such as the duration of field application or the cooling rate during field cooling can be expected to have an influence on the polarization as well as the piezoelectric behavior. ${ }^{10,11}$ When poling starts below the phase transition temperature, domain reversal is accomplished by the motion of domain walls. The velocity of these walls is proportional to the field applied. If the field is applied for a sufficiently long time, comparably high polarization can be achieved even with low fields. ${ }^{9}$ As the fieldcooling procedure takes longer when cooling from higher temperatures, a part of the increase in $d_{33}$ with increasing $T_{\text {start, }}$, as seen in Fig. 3(a), is possibly due to this effect of poling time. The predominant effect, however, will be the decrease in the poling field, as $d_{33}$ increases by more than two orders of magnitude from $T_{\text {start }}=100{ }^{\circ} \mathrm{C}$ to $T_{\text {start }}$ $=300{ }^{\circ} \mathrm{C}$, while the duration of the poling is increased by less than a factor of 3 . For very high poling temperatures, especially for $T_{\text {start }}>T_{\mathrm{C}}$, the time dependence of domainwall motion will cease to play a role, as domain walls are either very mobile and able to follow the external field nearly instantaneously or the domain structure is newly created when field cooling through the phase transition. An additional effect that might come into play in this case is the migration of mobile charge carriers, activated by the high temperature, which compensate for the depolarizing fields at domain walls and stabilize the domain structure. A similar effect has been observed when poling ferroelectric single crystals at high temperatures ${ }^{28,29}$ or when high fields are applied to a PZT ceramic at RT for long times. ${ }^{14}$ It is possible that due to this compensation for depolarizing fields, a stable macroscopic polarization can be achieved at high temperatures even for low poling fields, eliminating the field threshold observed in our experiments. However, the time scale required for this effect to be observed is outside the scope of the present investigation. Further investigations into this topic are currently being pursued.

\section{CONCLUSIONS}

Due to the increased domain mobility that results in lower coercive fields at high temperatures, field cooling is an effective way to obtain high piezoelectric coefficients with comparably low applied electrical field. However, depending on factors such as the nature of the phase transition or the polarization and strain at the transition point, there still is an energy threshold that must be overcome by the electric field to reach a maximum value of the polarization and $d_{33}$. Thus, effective poling cannot be achieved with arbitrarily low fields even when field cooling through the phase transition. In a temperature regime slightly below $T_{\mathrm{C}}$, a stable macroscopic remnant polarization cannot be obtained. This effect is most probably caused by stresses induced by polarization and strain mismatch at grain boundaries due to different crystallographic orientations of the grains and the distortion of the lattice structure at the transition. It makes the material appear macroscopically paraelectric, resulting in a source of 
error when measuring $T_{\mathrm{C}}$ by means that are susceptible only to long-range domain order. If this effect is taken into account, field cooling can be an effective method for the poling of piezoelectric ceramics.

\section{ACKNOWLEDGMENTS}

This work was supported by the Deutsche Forschungsgemeinschaft (Grant Nos. GR2722/4-1 and SFB 595).

${ }^{1}$ B. Jaffe, W. R. Cook, and H. Jaffe, Piezoelectric Ceramics (Academic, New York, 1971).

${ }^{2}$ A. J. Moulson and J. M. Herbert, Electroceramics: Materials Properties and Applications (Wiley, New York, 2003).

${ }^{3}$ K. H. Lam and H. L. W. Chan, Compos. Sci. Technol. 65, 1107 (2005).

${ }^{4}$ J. Juuti, H. Jantunen, V. P. Moilanen, and S. Leppavuori, J. Electroceram. 15, 57 (2005).

${ }^{5}$ H. Nagata and T. Takenaka, Jpn. J. Appl. Phys., Part 1 36, 6055 (1997).

${ }^{6}$ K. H. Lam, X. X. Wang, and H. L. W. Chan, Composites, Part A 36, 1595 (2005).

${ }^{7}$ Y. K. Gao, K. Uchino, and D. Viehland, J. Appl. Phys. 101, 054109 (2007).

${ }^{8}$ L. Wu and Y. Y. Lee, Jpn. J. Appl. Phys., Part 1 32, 5024 (1993).

${ }^{9}$ T. M. Kamel, F. X. N. M. Kools, and G. de With, J. Eur. Ceram. Soc. 27, 2471 (2007).

${ }^{10}$ M. Kohli and P. Muralt, Ferroelectrics 225, 155 (1999).

${ }^{11}$ T. M. Kamel and G. de With, J. Eur. Ceram. Soc. 28, 851 (2008).

${ }^{12}$ N. Balke, D. C. Lupascu, T. Granzow, and J. Rödel, J. Am. Ceram. Soc. 90, 1081 (2007).
${ }^{13}$ A. B. Kounga Njiwa, E. Aulbach, T. Granzow, and J. Rödel, Acta Mater. 55, 675 (2007).

${ }^{14}$ T. Granzow, A. B. Kounga, E. Aulbach, and J. Rödel, Appl. Phys. Lett. 88, 252907 (2006).

${ }^{15}$ H. Du, W. Zhou, F. Luo, D. Zhu, S. Qu, and Z. Pei, Appl. Phys. Lett. 91, 202907 (2007).

${ }^{16}$ K. Okazaki, Jpn. J. Appl. Phys., Part 1 32, 4241 (1993).

${ }^{17}$ K. Okazaki and H. Maiwa, Jpn. J. Appl. Phys., Part 1 31, 3113 (1992).

${ }^{18}$ C. A. Gallo and W. A. Schulze, J. Am. Ceram. Soc. 70, C33 (1987).

${ }^{19}$ M. Knapp, C. Baehtz, H. Ehrenberg, and H. Fuess, J. Synchrotron Radiat. 11, 328 (2004).

${ }^{20}$ M. Knapp, V. Joco, C. Baehtz, H. H. Brecht, A. Berghaeuser, H. Ehrenberg, H. von Seggern, and H. Fuess, Nucl. Instrum. Methods Phys. Res. A 521, 565 (2004).

${ }^{21}$ A. C. Larson and R. B. von Dreele, Los Alamos National Laboratory Report No. LAUR 86, 1994.

${ }^{22}$ K. A. Schönau, L. A. Schmitt, M. Knapp, H. Fuess, R.-A. Eichel, H. Kungl, and M. J. Hoffmann, Phys. Rev. B 75, 184117 (2007).

${ }^{23}$ An American National Standard, IEEE Standard on Piezoelectricity, IEEE Trans. Sonics Ultrason. 31, 1 (1984).

${ }^{24}$ S. N. Fedosov and H. von Seggern, J. Appl. Phys. 96, 2173 (2004).

${ }^{25}$ F. Jona and G. Shirane, Ferroelectric Crystals (Dover, New York, 1993), p. 20.

${ }^{26}$ W. Chang, A. H. King, and K. J. Bowman, Appl. Phys. Lett. 88, 242901 (2006).

${ }^{27}$ M. Liu, K. J. Hsia, and M. R. Sardela, J. Am. Ceram. Soc. 88, 210 (2005).

${ }^{28}$ T. Granzow, U. Dörfler, Th. Woike, M. Wöhlecke, R. Pankrath, M. Imlau, and W. Kleemann, Appl. Phys. Lett. 80, 470 (2002).

${ }^{29}$ T. Granzow, T. Woike, M. Wöhlecke, M. Imlau, and W. Kleemann, Phys. Rev. Lett. 89, 127601 (2002). 